\title{
Haemophilus parasuis invades porcine brain microvascular endothelial cells
}

\begin{abstract}
Correspondence
Marcelo Gottschalk

marcelo.gottschalk@umontreal.ca
\end{abstract}

Received 30 June 2005

Revised 7 September 2005

Accepted 4 October 2005

\section{Ghyslaine Vanier, ${ }^{1}$ Anna Szczotka, ${ }^{2}$ Peter Friedl, ${ }^{3}$ Sonia Lacouture, ${ }^{1}$ Mario Jacques ${ }^{1,2}$ and Marcelo Gottschalk ${ }^{1,2}$}

\author{
1,2Groupe de Recherche sur les Maladies Infectieuses du Porc (GREMIP) ${ }^{1}$ and Canadian \\ Research Network on Bacterial Pathogens of Swine ${ }^{2}$, Faculté de Médecine Vétérinaire, \\ Université de Montréal, Québec, Canada \\ ${ }^{3}$ Institute für Biochemie, Technische Hochschule Darmstadt, Petersenstrasse 22, \\ 64287 Darmstadt, Germany
}

\begin{abstract}
Haemophilus parasuis, an important swine pathogen, is the aetiological agent of Glässer's disease. It is responsible for cases of polyserositis, meningitis and pneumonia in young pigs. To date, 15 serotypes have been described, although several non-typable isolates are frequently recovered from diseased animals. The pathogenesis of $H$. parasuis infection is poorly understood. To cause meningitis, $H$. parasuis would have to cross the blood-brain barrier (BBB), composed of brain microvascular endothelial cells (BMEC). The objective of this study was to investigate the ability of $H$. parasuis to interact with porcine brain microvascular endothelial cells (PBMEC). It was demonstrated that the serotype 5 reference strain of $H$. parasuis, Nagasaki (originally recovered from a case of meningitis), was able to adhere at very high levels to and, most importantly, invade PBMEC. These capacities were confirmed by electron microscopy. Actinobacillus pleuropnemoniae serotype 7 (strain WF 83), used as negative control, was not able to adhere to or invade PBMEC. Comparisons of the levels of adhesion and invasion by several $H$. parasuis field strains from different serotypes isolated from cases of either meningitis or pneumonia showed that isolates of serotypes 4 and 5 had a higher invasion capacity than isolates belonging to other serotypes. Inhibition studies demonstrated that PBMEC invasion by $H$. parasuis required rearrangement of actin microfilaments and microtubular cytoskeletal elements but not active bacterial DNA, RNA or protein synthesis. Characterization studies demonstrated that proteinaceous invasin(s) does not seem to play a major role in entry of $H$. parasuis into PBMEC. Intracellular viable $H$. parasuis were found in PBMEC up to $6 \mathrm{~h}$ after antibiotic treatment. Even at high bacterial doses, $H$. parasuis was not toxic to PBMEC. In swine, the invasion of endothelial cells of the BBB may play an important role in the pathogenesis of meningitis caused by H. parasuis.
\end{abstract}

\section{INTRODUCTION}

Haemophilus parasuis, an important swine pathogen, is the aetiological agent of Glässer's disease. It is responsible for cases of polyserositis, meningitis and pneumonia in young pigs (Amano et al., 1994). To date, 15 serotypes have been described using an immunodiffusion test with heat-stable antigens (Kielstein et al., 1991; Morozumi \& Nicolet, 1986a;

Abbreviations: BBB, blood-brain barrier; BMEC, brain microvascular endothelial cells; CNS, central nervous system; LDH, lactate dehydrogenase; LOS, lipooligosaccharides; LPS, lipopolysaccharides; PBMEC, porcine brain microvascular endothelial cells; pPBMEC, primary porcine brain microvascular endothelial cells; PPLO, pleuropneumonia-like organisms; SEM, scanning electron microscopy; TEM, transmission electron microscopy.
Rapp-Gabrielson \& Gabrielson, 1992), although several non-typable isolates are frequently recovered from diseased animals (Rafiee \& Blackall, 2000). Recently, the use of the indirect haemagglutination test has been proposed by two studies in which less than $10 \%$ of non-typable strains were reported (Del Rio et al., 2003; Tadjine et al., 2004). However, Turni \& Blackall (2005) recommended the use of an indirect haemagglutination test only as a complement of the gel diffusion test in the case of a non-typable result or crossreaction. In North America, serotypes 4 and 5 are the most frequently isolated (Rapp-Gabrielson \& Gabrielson, 1992; Tadjine et al., 2004).

Virulence factors involved in $H$. parasuis infection are still unknown. The association of either capsule expression or whole-cell protein profiles with virulence of $H$. parasuis is 
controversial (Oliveira \& Pijoan, 2004). In addition, virulent and non-virulent strains share similar patterns of lipooligosaccharides (LOS) (Zucker et al., 1996) and the presence of fimbriae-like structures has been observed after in vivo passage (Munch et al., 1992). Finally, a neuraminidase activity has been described as a putative virulence factor (Lichtensteiger \& Vimr, 1997). It is hypothesized that this enzyme, in concert with permease and aldolase, may contribute to bacterial virulence by scavenging carbohydrates from host cells. However, the actual role of this enzyme in the persistence or virulence of $H$. parasuis has yet to be demonstrated. More recently, differential-display RT-PCR has been used to search for genes associated with virulence (Hill et al., 2003). Although some interesting candidate genes were found, no single virulence factor was clearly identified. It remains unclear if there is a relationship between the virulence of isolates and the serotype (Kielstein et al., 1991; Morozumi \& Nicolet, 1986a, b; Rapp-Gabrielson \& Gabrielson, 1992). However, it is generally acceptable to use serotype as a marker of pathogenicity (Oliveira \& Pijoan, 2004). In this way, serotypes $1,2,4,5,10,12,13,14$ and 15 are considered to be virulent while the other serotypes are thought to be avirulent (Amano et al., 1994; Kielstein \& Rapp-Gabrielson, 1992).

The pathogenesis of $H$. parasuis infection is poorly understood. The bacteria normally colonize the upper respiratory tract of pigs, which constitute their natural reservoir (Moller \& Kilian, 1990). Virulent strains of $H$. parasuis are able to breach the mucosal barrier and get into the bloodstream (Vahle et al., 1997). In vivo studies indicated that bacteria were not closely apposed to cilia or other cell structures (Vahle et al., 1997). However, no studies on the specific interactions between $H$. parsuis and host epithelial and/or endothelial cells have been performed.

Factors involved in systemic invasion by $H$. parasuis are unknown. To cause meningitis, $H$. parasuis has to reach the central nervous system (CNS). One way by which bacteria could gain access to CNS is to cross the blood-brain barrier (BBB). The BBB, an anatomically and functionally unique barrier, separates the CNS from the bloodstream and is responsible for maintaining homeostasis within the CNS. The BBB is formed, in part, by the endothelium lining the brain capillaries. This endothelium is composed of brain microvascular endothelial cells (BMEC) that form tight junctions regulating the traffic of solutes, macromolecules and cells across the BBB (Huang et al., 2000; Tuomanen, 1996). These microvessels are covered by pericytes and outgrowths of astrocytes (called astrocytic end-feet) (Goldstein \& Betz, 1986; Kniesel \& Wolburg, 2000). The primary site of breakdown of the BBB in most cases of bacterial meningitis appears to be the BMEC (Townsend \& Scheld, 1995). However, it is not clear how circulating $H$. parasuis crosses the BBB.

To further understand the pathogenesis of $H$. parasuisinduced meningitis in swine, the objective of this study was to investigate the capacity of $H$. parasuis to interact with porcine brain microvascular endothelial cells (PBMEC).

\section{METHODS}

Bacterial strains and growth conditions. The $H$. parasuis serotype 5 reference strain Nagasaki, isolated from a case of septicaemia with meningitis (Morozumi \& Nicolet, 1986b) and kindly provided by Dr R. F. Ross (Iowa State University), served as the reference strain. Other $H$. parasuis strains used in this study are listed in Table 1. Bacteria were grown for $48 \mathrm{~h}$ on PPLO agar plates (Difco) at $37^{\circ} \mathrm{C}$, and isolated colonies were inoculated in PPLO broth (Difco) for $8 \mathrm{~h}$ at $37^{\circ} \mathrm{C}$ with agitation. Working cultures were obtained by inoculating $10 \mu \mathrm{l}$ of a $10^{-3}$ dilution of these cultures in $30 \mathrm{ml}$ PPLO broth for $16 \mathrm{~h}$ at $37^{\circ} \mathrm{C}$ with agitation. Bacteria were washed twice in phosphate-buffered saline (PBS; $140 \mathrm{mM} \mathrm{NaCl}$, $3 \mathrm{mM} \mathrm{KCl}, 10 \mathrm{mM} \mathrm{Na}_{2} \mathrm{HPO}_{4}, 1 \cdot 5 \mathrm{mM} \mathrm{KH}_{2} \mathrm{PO}_{4}, \mathrm{pH} 7 \cdot 3$ ), and were appropriately diluted in cell culture medium before infection (see Results). An accurate determination of the number of c.f.u. per $\mathrm{ml}$ in the final suspension was made by plating onto PPLO agar supplemented with glucose $(0 \cdot 1 \%, \mathrm{w} / \mathrm{v})$ and $\beta$-nicotinamide adenine dinucleotide ( $\beta$-NAD; $0.004 \%$, w/v; Sigma). In addition, Actinobacillus pleuropneumoniae serotype 7 reference strain WF 83 was used as a negative control. A. pleuropneumoniae, which causes swine pleuropneumonia, is very rarely found in the blood circulation and is not known to cause meningitis (Gottschalk \& Taylor, 2005).

Cell culture. The porcine brain microvascular endothelial cell line PBMEC/C1-2 (Teifel \& Friedl, 1996) was cultivated as previously described (Vanier et al., 2004). Briefly, cells were grown in complete

Table 1. Strains used in this study

\begin{tabular}{|lcll|}
\hline Strain & Serotype & Pathology & Provided by $\dagger$ \\
\hline Nagasaki* $^{\star}$ & 5 & Meningitis & R. F. Ross \\
KBQ N1Br & 5 & Meningitis & C. Pijoan \\
KBQ WBr1 & 5 & Meningitis & C. Pijoan \\
Pig 9-Y & 4 & Meningitis & C. Pijoan \\
34/6 156 & 4 & Meningitis & C. Pijoan \\
46252-Y & 2 & Meningitis & C. Pijoan \\
35594 Bre-Y & 1 & Meningitis & C. Pijoan \\
Pig 11-Y & 12 & Meningitis & C. Pijoan \\
Pig 27 (4 weeks in)-Y & 12 & Meningitis & C. Pijoan \\
03-2402 A & 7 & Meningitis & C. Pijoan \\
03-001198-Y & 7 & Meningitis & C. Pijoan \\
STF 04-0507-2 & 5 & Pneumonia & K. R. Mittal \\
STF 04-0348 & 4 & Pneumonia & K. R. Mittal \\
STF 03-6521-2 & 4 & Pneumonia & K. R. Mittal \\
STF 04-0116 & 4 & Pneumonia & K. R. Mittal \\
STF 03-6681 & 2 & Pneumonia & K. R. Mittal \\
STF 03-6562 & 2 & Pneumonia & K. R. Mittal \\
STF 03-6369-1 & 4 & Pneumonia & K. R. Mittal \\
STF 03-6900 & 7 & Pneumonia & K. R. Mittal \\
STF 03-7961 & 12 & Pneumonia & K. R. Mittal \\
SHY 03-4432 & 13 & Pneumonia & K. R. Mittal \\
& & & \\
\hline Refen & & & \\
\hline
\end{tabular}

${ }^{*}$ Reference strain used in this study.

$\dagger$ R. F. Ross, Iowa State University; C. Pijoan, University of Minnesota; K. R. Mittal, Université de Montréal. 
IF medium, which is a mixture of $1: 1$ Iscove's modified Dulbecco's medium and Ham's F-12 (Invitrogen) supplemented with $7 \cdot 5 \%$ $(\mathrm{v} / \mathrm{v})$ heat-inactivated foetal bovine serum (hiFBS), penicillinstreptomycin (Invitrogen), sodium bicarbonate, L-glutamine, human transferrin (MP Biomedicals), $\mathrm{N}$-acetylcysteine, hypoxanthine, porcine heparin, human recombinant fibroblast growth factor-basic (Sigma), and $\beta$-mercaptoethanol (Bio-Rad). Flasks (Falcon; Becton Dickinson) and Primaria 24-well tissue culture plates (Falcon) were precoated with $1 \%(\mathrm{w} / \mathrm{v})$ type A gelatin from porcine skin (Sigma) to support the cells. Cells were incubated at $37^{\circ} \mathrm{C}$ with $5 \% \mathrm{CO}_{2}$ in a humid atmosphere and used before the 20th passage for all experiments. Primary porcine brain microvascular endothelial cells (pPBMEC) were purchased from Cell Systems and were grown according to the supplier's recommendations. For assays, PBMEC and pPBMEC were trypsinized and diluted in their culture medium at $1 \times 10^{5}$ cells $\mathrm{ml}^{-1}$. The cell suspension was distributed in tissue culture plates and incubated to confluence. Immediately before the experiments, medium was removed from the plates and replaced by the respective medium without antibiotics.

PBMEC adhesion and invasion assays. The adhesion assay to quantify total cell-associated (intracellular plus surface-adhered) bacteria was performed as previously described (Vanier et al., 2004), with some modifications. Briefly, bacteria were pelleted, washed twice with PBS, and resuspended in fresh cell culture medium without antibiotics at $10^{7}$ c.f.u. $\mathrm{ml}^{-1}$. Confluent monolayers of PBMEC grown in 24-well plates were infected with $1 \mathrm{ml}$ aliquots of bacterial suspension. The plates were incubated for different times up to $2 \mathrm{~h}$ at $37^{\circ} \mathrm{C}$ to allow bacterial adhesion. Thereafter, cells were vigorously washed five times with $\mathrm{PBS}$ to eliminate non-specific bacterial attachment and incubated for $10 \mathrm{~min}$ at $37^{\circ} \mathrm{C}$ in the presence of $200 \mu \mathrm{l} 0.05 \%$ trypsin $/ 0.03 \%$ EDTA. After this incubation period, $800 \mu \mathrm{l}$ ice-cold deionized water was added, and cells were disrupted by scraping the bottom of the well and by repeated pippeting to liberate cell-associated bacteria. Serial dilutions of this cell lysate were plated onto PPLO agar and incubated for $48 \mathrm{~h}$ at $37^{\circ} \mathrm{C}$. Levels of adhesion are expressed as the total number of c.f.u. recovered per well.

The invasion assay to quantify intracellular bacteria was performed in a similar manner to the adhesion assay, but the plates were centrifuged at $800 \mathrm{~g}$ for $10 \mathrm{~min}$ to bring bacteria to the surface of the monolayer and incubated for different times up to $4 \mathrm{~h}$. The monolayers were then washed four times with PBS; $1 \mathrm{ml}$ cell culture medium containing $100 \mu \mathrm{g}$ gentamicin $\mathrm{ml}^{-1}$ and $5 \mu \mathrm{g}$ penicillin $\mathrm{G} \mathrm{ml}^{-1}$ (Sigma) was added to each well, and the plates were incubated for $2 \mathrm{~h}$ at $37^{\circ} \mathrm{C}$ to kill extracellular and surface-adhered bacteria. The monolayers were washed three times with PBS before incubation with $0.05 \%$ trypsin/ $0.03 \%$ EDTA. Controls using a bacterial concentration similar to the resulting concentration of surface-adhered bacteria were performed to ensure that the antibiotic concentrations used were sufficient to kill all extracellular cell-associated bacteria after $2 \mathrm{~h}$ of treatment. Adhesion and invasion assays were performed under non-toxic conditions (see below). For the intracellular survival assay, an invasion assay was performed as described above except that after a $2 \mathrm{~h}$ invasion period, the initial gentamicin/penicillin G treatment was lengthened for different times up to an additional $6 \mathrm{~h}$. Data are expressed as the total number of recovered c.f.u. per well after antibiotic treatment.

Invasion inhibition studies. For experiments testing the effects of inhibitors of bacterial DNA, RNA and protein synthesis, bacteria were pre-treated with ciprofloxacin (Bayer), rifampicin (Sigma) or tetracycline (Sigma), respectively. $H$. parasuis strain Nagasaki $\left(10^{7}\right.$ c.f.u. $\left.\mathrm{ml}^{-1}\right)$ was treated with the appropriate concentration of inhibitor at $37^{\circ} \mathrm{C}$ for $30 \mathrm{~min}$ prior to infection, as well as during the $2 \mathrm{~h}$ invasion period. Respective MICs (determined according to NCCLS recommendations) for strain Nagasaki were
$<0 \cdot 125 \mu \mathrm{g} \mathrm{ml}^{-1}$ for ciprofloxacin, $0 \cdot 125 \mu \mathrm{g} \mathrm{ml}^{-1}$ for rifampicin and $0.25 \mu \mathrm{g} \mathrm{ml}^{-1}$ for tetracycline. In addition to MICs, concentrations corresponding to one-half and twice the MICs were also used for each antibiotic. Results are expressed as percentage invasion relative to the invasion level without inhibitor (taken as $100 \%$ invasion).

To study the effect of proteases affecting outer-surface proteins, $H$. parasuis strain Nagasaki was treated with proteinase K (Roche) at $0 \cdot 1-2 \mathrm{mg} \mathrm{ml}^{-1}$ for $1 \mathrm{~h}$ at $37^{\circ} \mathrm{C}$, washed twice in PBS, resuspended in culture medium, and used to infect PBMEC for $2 \mathrm{~h}$.

For experiments testing the effects of inhibitors of the formation of microfilaments and microtubules, PBMEC monolayers were preincubated with cytochalasin D or colchicine (Sigma), respectively. Different concentrations of the inhibitors were added at $37^{\circ} \mathrm{C}$ for $30 \mathrm{~min}$ prior to the infection with strain Nagasaki, as well as during the $2 \mathrm{~h}$ invasion period. Cytochalasin $\mathrm{D}$ concentrations of $0 \cdot 25,0.5,1 \cdot 0$ and $2 \cdot 0 \mu \mathrm{g} \mathrm{ml}^{-1}$ and colchicine concentrations of $1 \cdot 0,5 \cdot 0,10$ and $20 \mu \mathrm{g} \mathrm{ml}^{-1}$ were used. Results are expressed as percentage invasion relative to invasion level without inhibitor (taken as $100 \%$ invasion).

PBMEC cytotoxicity assay. The cytotoxic effect of bacteria was evaluated by lactate dehydrogenase (LDH) measurement. Briefly, bacteria were grown and diluted as described above and confluent monolayers of PBMEC grown in 24-well plates were infected with $1 \mathrm{ml}$ aliquots of bacterial suspension. The plates were centrifuged at $800 \mathrm{~g}$ for $10 \mathrm{~min}$ to bring bacteria to the surface of the monolayer. The plates were incubated at $37^{\circ} \mathrm{C}$ for different times up to $18 \mathrm{~h}$. Non-infected cells and bacteria in IF medium without a PBMEC monolayer were used as negative controls, whereas cells lysed in $0.025 \%$ Triton X-100 were used as a positive control ( $100 \%$ toxicity). At the end of the incubation period, a $100 \mu \mathrm{l}$ aliquot of each supernatant was transferred to a 96-well plate, which was centrifuged at $250 \mathrm{~g}$ for $5 \mathrm{~min}$. LDH measurement was performed on $50 \mu \mathrm{l}$ aliquots of each centrifuged supernatant using the CytoTox 96 nonradioactive cytotoxicity assay (Promega).

Electron microscopy studies. For transmission electron microscopy (TEM), PBMEC cell monolayers were grown on $13 \mathrm{~mm}$ Thermanox coverslips in a 24-well culture plate. Adhesion and invasion assays were performed as described above. After two washes with PBS, the monolayers were fixed for $1 \mathrm{~h}$ at room temperature with $2 \%(\mathrm{v} / \mathrm{v})$ glutaraldehyde in $0 \cdot 1 \mathrm{M}$ cacodylate buffer $\mathrm{pH} 7 \cdot 3$ and then samples were postfixed in $2 \%(\mathrm{v} / \mathrm{v})$ osmium tetroxide in deionized water. Specimens were dehydrated in a graded series of ethanol solutions and embedded in Spurr resin. Thin sections were cut with a diamond knife on a Leica Ultracut ultramicrotome and post-stained with uranyl acetate and lead citrate. Samples were observed with a model 420 electron microscope (Philips Electronics). For scanning electron microscopy (SEM), samples were processed as for the TEM, except that they were not postfixed with osmium tetroxide. Samples for SEM were dehydrated in a graded series of ethanol solutions and covered with gold after critical-point drying and examined using a Hitachi S-3000N microscope.

Statistical analysis. All data are expressed as means \pm standard deviations (error bars). Data were analysed by two-tailed, unpaired $t$ test. For comparison of adhesion and invasion capacities of several $H$. parasuis strains, a linear mixed model followed by Tukey-Kramer post hoc test were used. A $P$ value of $<0.05$ was considered significant. All assays were repeated at least three times in independent experiments. 


\section{RESULTS}

\section{High bacterial doses of $\boldsymbol{H}$. parasuis are not toxic to PBMEC}

To determine whether $H$. parasuis is cytotoxic to PBMEC, LDH release measurements were performed. During the $18 \mathrm{~h}$ incubation period, less than $2 \%$ cytotoxicity was observed with strain Nagasaki even at a high concentration of $10^{8}$ c.f.u. $\mathrm{ml}^{-1}$. Based on these results, the bacterial concentration used for the $2 \mathrm{~h}$ invasion assay $\left(10^{7}\right.$ c.f.u. $\left.\mathrm{ml}^{-1}\right)$ described above was not deemed to be toxic to the cells. A. pleuropneumoniae strain WF 83 gave a good positive control, since it exhibited $22 \% \pm 5 \%$ toxicity after $4 \mathrm{~h}$ of incubation and toxicity increased considerably after $6 \mathrm{~h}$ of incubation, to reach $88 \% \pm 14 \%$. As was the case for $H$. parasuis, conditions used for adhesion/invasion assays with this strain were not cytotoxic.

\section{H. parasuis adhesion to PBMEC}

$H$. parasuis was able to adhere to PBMEC at high levels. This adhesion was time-dependent (Fig. 1a) and reached a plateau after $30 \mathrm{~min}$ incubation. The time-dependent adhesion kinetics was not due to an increase in the number of c.f.u. since $H$. parasuis did not grow during the $2 \mathrm{~h}$ incubation period used for adhesion assay (data not shown). Also, adhesion was dependent on the concentration of bacteria (Fig. 1b). A. pleuropneumoniae strain WF 83 was
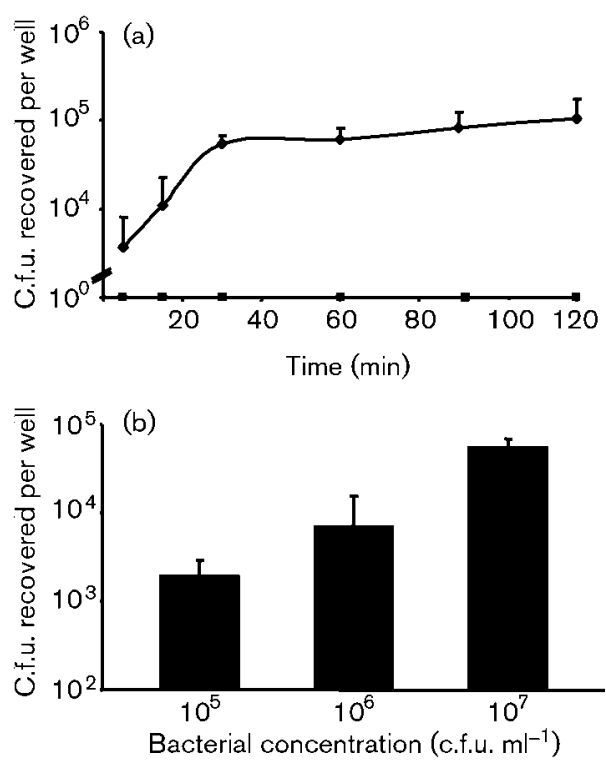

Fig. 1. (a) Kinetics of $H$. parasuis adhesion to PBMEC. PBMEC were exposed to $H$. parasuis $(\boldsymbol{)})$ or $A$. pleuropneumoniae $(\boldsymbol{\square})$ for the indicated times and then washed extensively to remove nonadherent bacteria. Cell lysates were plated onto PPLO agar to obtain bacterial viable plate counts as described in Methods. (b) Effect of $H$. parasuis concentration on level of adhesion to PBMEC after 30 min using the protocol described above. used as negative control for the adhesion assays and no bacteria were recovered throughout the $2 \mathrm{~h}$ invasion period (Fig. 1a).

\section{PBMEC invasion by $\boldsymbol{H}$. parasuis}

$H$. parasuis was able to invade PBMEC and this invasion capacity depended on incubation time (Fig. 2a) and bacterial concentration (Fig. 2b). No significant increase in invasion was observed after $1 \mathrm{~h}$ incubation $(P>0 \cdot 05) . A$. pleuropneumoniae strain WF 83 was used as a negative control for the invasion assays and no bacteria were recovered throughout the $4 \mathrm{~h}$ invasion period (Fig. 2a).

To confirm that $H$. parasuis adhesion to and invasion of PBMEC was not due to cell immortalization, primary PBMEC were used. Similar levels of adhesion and invasion were observed between pPBMEC and immortalized PBMEC (data not shown).

\section{Confirmation of adhesion and invasion by electron microscopy}

TEM and SEM were used to confirm adhesion and invasion. After 15 min incubation, bacteria were observed to adhere to PBMEC (Fig. 3a). Fig. 3(b) shows $H$. parasuis bacteria in close contact with a cell and within an invagination.
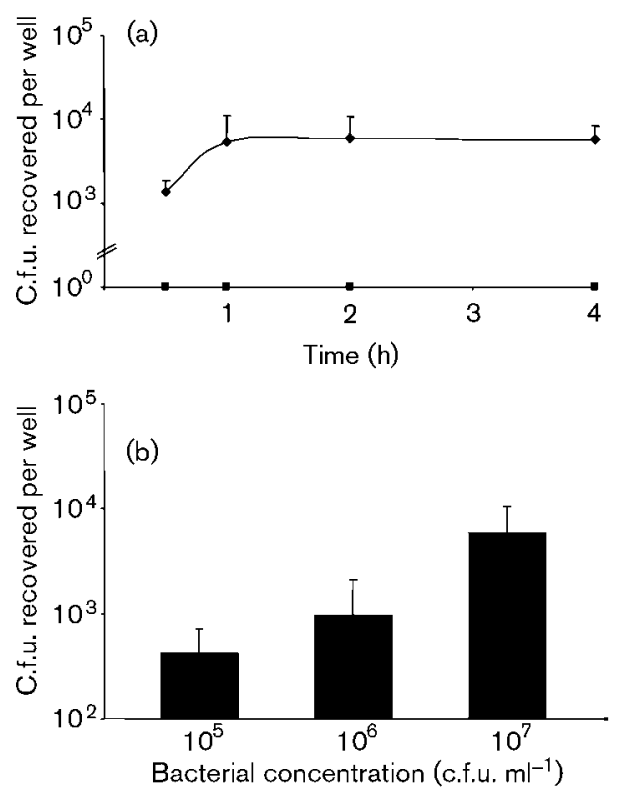

Fig. 2. (a) Kinetics of PBMEC invasion by $H$. parasuis. PBMEC were exposed to $H$. parasuis $(\boldsymbol{})$ or $A$. pleuropneumoniae ( $\boldsymbol{\square})$, then washed extensively to remove nonadherent bacteria and treated with antibiotics to kill extracellular bacteria, followed by further washing and lysis of the PBMEC. Cell lysates were plated onto PPLO agar to obtain bacterial viable plate counts as described in Methods. (b) Effect of $H$. parasuis concentration on level of PBMEC invasion after $2 \mathrm{~h}$ using the protocol described above. 

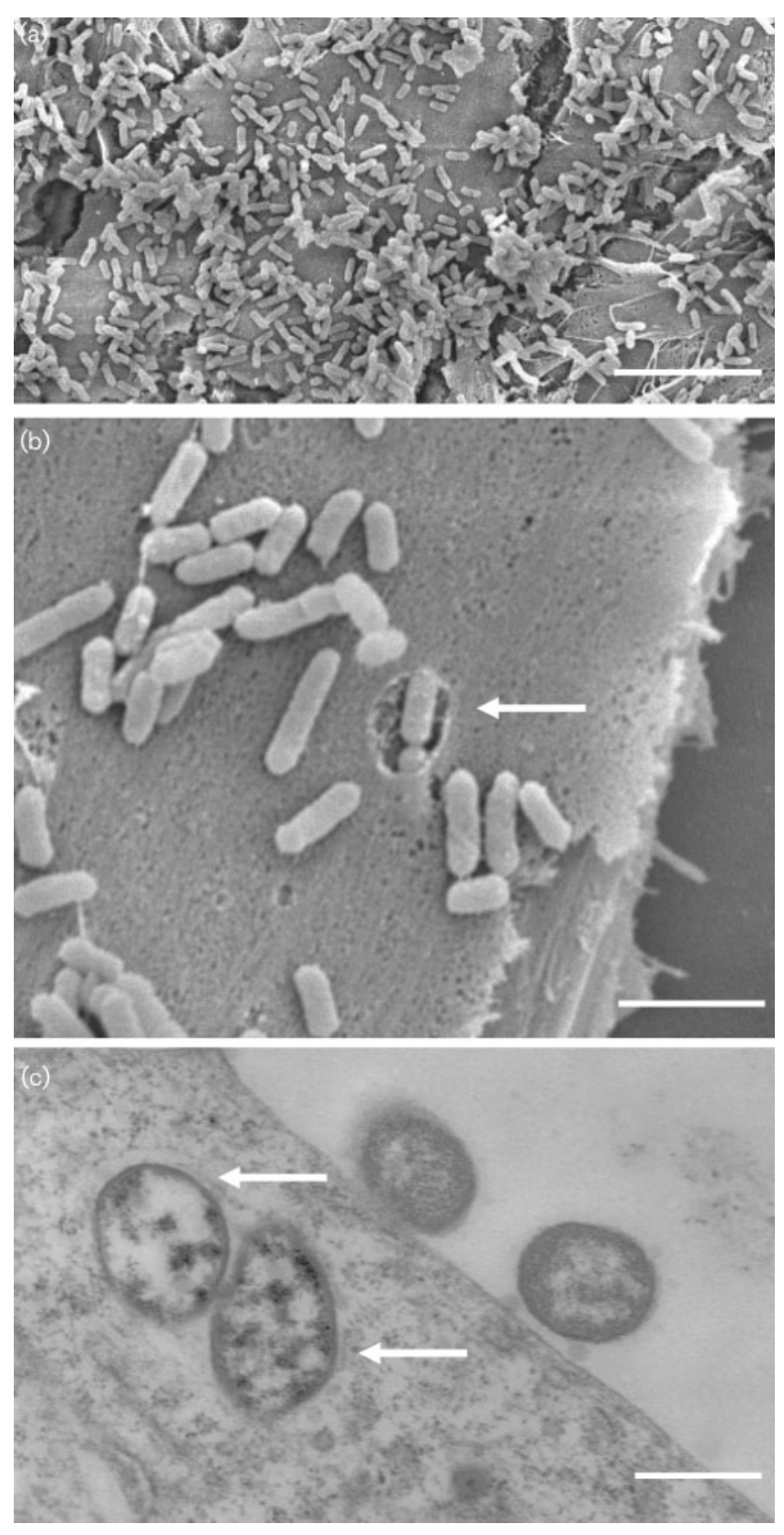

Fig. 3. SEM (a, b) and TEM (c) micrographs showing $H$. parasuis interactions with PBMEC after $15 \mathrm{~min}(\mathrm{a}, \mathrm{b})$ and $2 \mathrm{~h}$ (c) incubation. The arrows indicate invading $H$. parasuis. Bars: $8 \mu \mathrm{m}$ (a), $2 \mu \mathrm{m}$ (b), $0.5 \mu \mathrm{m}$ (c).

Fig. 3(c) provides evidence confirming the intracellular invasion of PBMEC by $H$. parasuis.

\section{H. parasuis intracellular survival within PBMEC}

As shown in Fig. 4, after an initial $2 \mathrm{~h}$ invasion period and a minimal $2 \mathrm{~h}$ treatment with antibiotics, viable $H$. parasuis strain Nagasaki were found inside PBMEC up to an additional $6 \mathrm{~h}$ of incubation in the presence of antibiotics to exclude any extracellular source of bacteria. The results

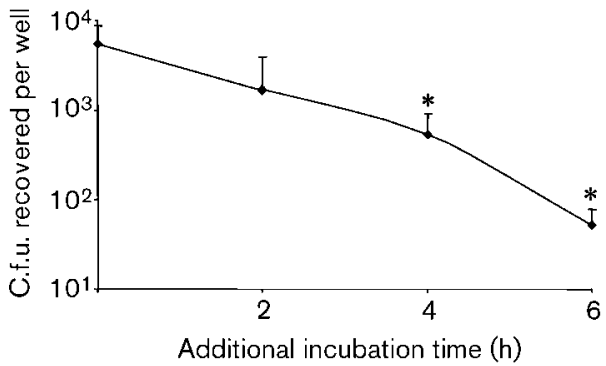

Fig. 4. Intracellular survival of $H$. parasuis $\left(10^{7}\right.$ c.f.u. $\left.\mathrm{ml}^{-1}\right)$ within PBMEC. Additional incubation time represents time after the standard $2 \mathrm{~h}$ antibiotic treatment of the invasion assay. An asterisk indicates a significant difference $(P<0.05)$ between the number of intracellular bacteria recovered per well obtained at the additional incubation time and the number obtained after the initial $2 \mathrm{~h}$ antibiotic treatment (time $0 \mathrm{~h}$ ).

showed a gradual decrease in the number of viable intracellular bacteria, which is significant $(P<0.05)$ after $4 \mathrm{~h}$ of incubation with antibiotics.

\section{Comparison of adhesion and invasion capacities of several field $\boldsymbol{H}$. parasuis strains}

Several field $H$. parasuis strains were compared for their ability to adhere to and invade PBMEC according to their serotype and the pathology (meningitis or pneumonia) observed in the infected pigs from which they were originally isolated. Our results showed that the ability of $H$. parasuis to adhere to and invade PBMEC was observed not only with the reference strain Nagasaki but also with most of the field strains tested. Although individual differences were observed in levels of adhesion and invasion among the strains, no correlation was found with the pathology (meningitis or pneumonia) caused by the strains (data not shown). On the other hand, the mean capacity to adhere to PBMEC was similar among all serotypes $(1,2,4,5,7,12$ and $13)$ tested $(P>0 \cdot 05$; data not shown). However, differences in mean invasion levels were observed among serotypes. Although only a few strains of some serotypes were tested, strains of serotypes 4 and 5 exhibited a higher invasion capacity than strains belonging to other serotypes (data not shown). If the mean invasion result for serotype 5 is considered as $100 \%$, mean invasion results are $130 \%, 34 \%$, $14 \%, 9 \%, 9 \%$ and $0 \cdot 2 \%$ for serotypes $4,2,7,1,12$ and 13 , respectively.

\section{Characterization of PBMEC invasion by H. parasuis}

To evaluate the possible role of bacterial DNA, RNA and protein synthesis in invasion of PBMEC by $H$. parasuis, bacteria were pre-treated with ciprofloxacin, rifampicin or tetracycline. When used at their respective MICs, none of these antibiotics was able to inhibit PBMEC invasion by $H$. parasuis strain Nagasaki (data not shown). Similar levels of 
invasion were observed using concentrations corresponding to one-half and twice the MICs for all antibiotics (data not shown).

To determine whether bacterial proteins play a role in the invasion of PBMEC, bacteria were pretreated with proteinase $\mathrm{K}$ at a maximal concentration of $2 \mathrm{mg} \mathrm{ml}^{-1}$ that did not exert any effect on the viability of the bacteria (data not shown). The treatment did not affect invasion, as demonstrated by the similar level of invasion $(94 \% \pm 22 \%)$ by treated bacteria as that by non-treated bacteria (taken as $100 \%)$. Parallel studies using an invasive strain of Streptococcus suis showed that the proteinase $\mathrm{K}$ treatment was effective since it significantly affected the invasion capacity of this bacterial species (data not shown).

To evaluate the possible role of microfilaments and microtubules in invasion of PBMEC by $H$. parasuis, cells were pre-treated with cytochalasin $\mathrm{D}$ or colchicine, respectively. As shown in Fig. 5, both cytochalasin D and colchicine inhibited the invasion of PBMEC by $H$. parasuis strain Nagasaki. A cytochalasin D concentration of $2 \mu \mathrm{g} \mathrm{ml}^{-1}$ inhibited more than $80 \%$ of invasion $(P<0 \cdot 05)$, while $20 \mu \mathrm{g}$ colchicine $\mathrm{ml}^{-1}$ was able to inhibit more than $70 \%$ of invasion $(P<0 \cdot 05)$. None of the products used as eukaryotic inhibitors of PBMEC invasion was shown to be toxic to the PBMEC or bacteria at the concentrations used (data not shown).

\section{DISCUSSION}

Although $H$. parasuis is one of the most important swine pathogens worldwide, very little is known about the virulence factors or markers. In addition, there are currently no vaccines available that effectively protect animals against the infection. However, a study by Oliveira et al. (2001) showed that experimental inoculation of 5-day-old piglets with a live systemic strain of $H$. parasuis may be more effective

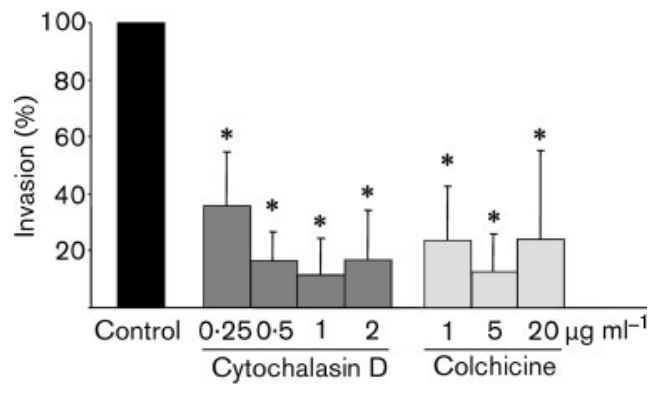

Fig. 5. Inhibition of invasion of PBMEC by $H$. parasuis strain Nagasaki $\left(10^{7}\right.$ c.f.u. $\left.\mathrm{ml}^{-1}\right)$ with cytochalasin $\mathrm{D}$ and colchicine. PBMEC monolayers were preincubated with the indicated concentrations of inhibitors at $37^{\circ} \mathrm{C}$ for 30 min prior to infection as well as during the $2 \mathrm{~h}$ invasion period. An asterisk indicates a significant difference $(P<0.05)$ in comparison with the level of invasion without inhibitor (taken as $100 \%$ ). in reducing morbidity and mortality than the colonization of piglets by nose-to-nose contact with inoculated sows. The pathogenesis of $H$. parasuis infection is also poorly understood. Increasing our understanding of the mechanisms by which these bacteria cause disease may help advance the development of effective tools to control the infection.

Of the many clinical diseases arising from $H$. parasuis infection, meningitis is one of the most frequently observed complications. To enter the CNS and cause disease, circulating $H$. parasuis has to first resist phagocytosis in order to remain at high concentrations in the bloodstream. In the only study published to date, Segales et al. (1998) reported that $H$. parasuis is able to resist phagocytosis by alveolar macrophages. In fact, the notion that a capsular polysaccharide is involved in protecting bacteria from phagocytosis is still controversial (Oliveira \& Pijoan, 2004). Once in the bloodstream, bacteria must be able to cross the BBB in order to reach the CNS and cause meningitis. Since BMEC are the most important components of the BBB, in this study we investigated the interactions between $H$. parasuis and PBMEC.

High numbers of $H$. parasuis were observed to adhere to PBMEC in a time-dependent manner. This finding is in agreement with the time-dependent adhesion of Streptococcus suis, another swine meningeal pathogen, to PBMEC (Vanier et al., 2004). However, H. parasuis appears to possess a considerably higher capacity than $S$. suis to adhere to these cells. Indeed, adhesion to PBMEC by $H$. parasuis reached a plateau (at $30 \mathrm{~min}$; this study) more rapidly than the adhesion by S. suis (at 4 to $6 \mathrm{~h}$; Vanier et al., 2004). In contrast to the experiments with S. suis, results in this study were obtained without spinning down bacteria to facilitate contact with PBMEC. In fact, almost $100 \%$ adhesion was obtained immediately when $H$. parasuis was spun down with the cells (unpublished observations). The high capacity of $H$. parasuis to adhere to PBMEC was also observed by electron microscopy.

In addition to adhesion, this study demonstrated that $H$. parasuis is also able to invade PBMEC. To the best of our knowledge, this is the first report on $H$. parasuis invasion of endothelial cells. Invasion of PBMEC was confirmed by SEM and TEM. Invasion of BMEC by free bacteria has also been reported for other Gram-negative meningeal pathogens, such as Haemophilus influenzae (Daines et al., 2003), Escherichia coli K1 (Badger \& Kim, 1998) and Citrobacter freundii (Badger et al., 1999). Our results showed that binding to and entry of $H$. parasuis into PBMEC were not due to immortalization of PBMEC since $H$. parasuis was able to invade primary PBMEC at levels similar to those observed with immortalized cells. Interestingly, A. pleuropneumoniae, a respiratory pathogen that does not cause systemic disease, was unable to adhere to or invade either type of cells.

Results in this study suggest that active bacterial DNA, RNA and protein synthesis may not be required by $H$. parasuis to invade PBMEC. These findings are consistent with those 
reported by Virji et al. (1991) on HUVEC invasion by Haemophilus influenzae. Thus, the entry of the bacterium may be mediated by pre-existing putative invasin(s) interacting with a cell receptor (Huang \& Jong, 2001; Townsend \& Scheld, 1995). In addition, proteinaceous invasin(s) does not seem to play a major role in $H$. parasuis invasion. However, the possibility that some surface proteins that might also contribute to the invasion process could have been restored after the $2 \mathrm{~h}$ incubation period of invasion can not completely be ruled out. It may be hypothesized that other non-proteinaceaous surface components, such as LOS, could play an important role in $H$. parasuis adhesion and entry into PBMEC. LPS from $A$. pleuropneumoniae were shown to be involved in adherence to porcine respiratory tract cells (Belanger et al., 1990; Jacques et al., 1991). Studies on the possible role of LOS in the interactions between $H$. parasuis and endothelial cells are warranted.

Reorganization of host cytoskeleton components, namely actin microfilaments and microtubules, was observed to be required for entry of $H$. parasuis into PBMEC. In agreement with our findings, the invasion of Chang epithelial cells by H. influenzae (St Geme \& Falkow, 1990) and of bovine aortic endothelial cells by Pasteurella multocida (Galdiero et al., 2001) was found to be dependent on microfilament and microtubule formation. Thus, internalization of other members of the family Pasteurellaceae may involve similar microfilament and microtubule reorganizations.

It has been suggested that many invading pathogens enter endothelial cells as a transient process (Hippenstiel \& Suttorp, 2003). This is likely to be the case for $H$. parasuis since the intracellular bacteria survive inside PBMEC but their number decreases gradually with time. It can be hypothesized that $H$. parasuis undergoes exocytosis after invasion as described for other pathogens, such as $P$. multocida with bovine aortic cells (Galdiero et al., 2001). However, degraded $H$. parasuis bacteria were exceptionally observed inside PBMEC by TEM (data not shown), indicating that the decreasing number of live intracellular bacteria could also be due in part to bacterial death. In vitro BBB models should be used to confirm the proposed hypothesis.

Cell adherence and subsequent entry of the bacteria into PBMEC were not associated with pathology caused by the strains. A previous study by Rapp-Gabrielson et al. (1992) showed that following an intraperitoneal inoculation, $H$. parasuis strains of serotypes 2,3 and 5 could be isolated from nearly all cultured tissues, including brain and lungs. Although no differences were observed in levels of adhesion, strains of serotypes 4 and 5 exhibited a higher level of invasion than strains of other serotypes. Interestingly, serotypes 4 and 5 are the most prevalent types isolated from diseased pigs in North America (Rapp-Gabrielson \& Gabrielson, 1992). However, since only a few strains of some serotypes were used in this study, conclusions are limited. A study including a large number of strains from all known serotypes would confirm this hypothesis.
It has been previously suggested that $H$. parasuis is able to damage the mucosal epithelium and induce inflammation through the release of one or more toxic compounds (Vahle et al., 1997). Interestingly, $H$. parasuis does not posses Apx toxin genes, in contrast to other members of the family Pasteurellaceae such as A. pleuropneumoniae and Actinobacillus suis (Schaller et al., 2000). However, direct studies on the toxic effect of $H$. parasuis on host cells have not been performed thus far. In the present study, using a highly sensitive test, $H$. parasuis exerted no detectable toxic activity toward PBMEC even though it was expected that high concentrations of LOS from $H$. parasuis would be toxic to the cells. It has been shown that whole cells and LPS from $H$. influenzae are able to disrupt a monolayer of bovine BMEC (Patrick et al., 1992). However, this toxic activity appears to be specific to the origin of the cells since another study, using rat BMEC, showed an increase of albumin permeability of the $\mathrm{BBB}$ in the absence of cell death as assessed by LDH release (Tunkel et al., 1991). Similarly, a possible role of $H$. parasuis and/or its LOS in potentially increasing permeability of BBB with swine cells cannot be ruled out. In fact, a role of endotoxin in the pathogenicity of $H$. parasuis in acute septicaemia in pigs has been reported (Amano et al., 1997). Cell toxicity observed with A. pleuropneumoniae serotype 7 after prolonged incubation was probably due to the presence of ApxII toxin (Choi et al., 2001).

In conclusion, this study is the first to describe a high capacity of $H$. parasuis to adhere to and invade PBMEC. Further studies are needed to characterize the molecule(s) that mediate the adherence to and/or invasion of PBMEC. In swine, the entry of $H$. parasuis into endothelial cells constituting the BBB may be a key step in the pathogenesis of meningitis caused by this pathogen.

\section{ACKNOWLEDGEMENTS}

We thank D. Meunier-Côté for technical assistance. We also acknowledge D. Montpetit from the Centre de Recherche et Développement sur les Aliments (CRDA) for the transmission and scanning electron microscopy. We are indebted to Dr K. R. Mittal and Dr C. Pijoan for providing some of the strains used in this study. This work was supported by the Canadian Research Network on Bacterial Pathogens of Swine (CRNBPS) and the Fonds québécois de la recherche sur la nature et les technologies (FQRNT) grant no. PR106088. G. V. is recipient of an NSERC scholarship.

\section{REFERENCES}

Amano, H., Shibata, M., Kajio, N. \& Morozumi, T. (1994). Pathologic observations of pigs intranasally inoculated with serovar 1, 4 and 5 of Haemophilus parasuis using immunoperoxidase method. J Vet Med Sci 56, 639-644.

Amano, H., Shibata, M., Takahashi, K. \& Sasaki, Y. (1997). Effects on endotoxin pathogenicity in pigs with acute septicemia of Haemophilus parasuis infection. J Vet Med Sci 59, 451-455.

Badger, J. L. \& Kim, K. S. (1998). Environmental growth conditions influence the ability of Escherichia coli $\mathrm{K} 1$ to invade brain microvascular endothelial cells and confer serum resistance. Infect Immun 66, 5692-5697. 
Badger, J. L., Stins, M. F. \& Kim, K. S. (1999). Citrobacter freundii invades and replicates in human brain microvascular endothelial cells. Infect Immun 67, 4208-4215.

Belanger, M., Dubreuil, D., Harel, J., Girard, C. \& Jacques, M. (1990). Role of lipopolysaccharides in adherence of Actinobacillus pleuropneumoniae to porcine tracheal rings. Infect Immun 58, 3523-3530.

Choi, C., Kwon, D., Min, K. \& Chae, C. (2001). Detection and localization of ApxI, -II and -III genes of Actinobacillus pleuropneumoniae in natural porcine pleuropneumonia by in situ hybridization. Vet Pathol 38, 390-395.

Daines, D. A., Cohn, L. A., Coleman, H. N., Kim, K. S. \& Smith, A. L. (2003). Haemophilus influenzae Rd KW20 has virulence properties. J Med Microbiol 52, 277-282.

Del Rio, M. L., Gutierrez, C. B. \& Rodriguez Ferri, E. F. (2003). Value of indirect hemagglutination and coagglutination tests for serotyping Haemophilus parasuis. J Clin Microbiol 41, 880-882.

Galdiero, M., De Martino, L., Pagnini, U., Pisciotta, M. G. \& Galdiero, E. (2001). Interactions between bovine endothelial cells and Pasteurella multocida: association and invasion. Res Microbiol 152, 57-65.

Goldstein, G. W. \& Betz, A. L. (1986). The blood-brain barrier. Sci Am 255, 74-83.

Gottschalk, M. \& Taylor, D. J. (2005). Actinobacillus pleuropneumoniae. In Diseases of Swine, Edited by B. E. Straw, S. D'Allaire, W. L. Mengeling \& D. J. Taylor. Ames: Iowa State University (in press).

Hill, C. E., Metcalf, D. S. \& Maclnnes, J. I. (2003). A search for virulence genes of Haemophilus parasuis using differential display RT-PCR. Vet Microbiol 96, 189-202.

Hippenstiel, S. \& Suttorp, N. (2003). Interaction of pathogens with the endothelium. Thromb Haemost 89, 18-24.

Huang, S. H. \& Jong, A. Y. (2001). Cellular mechanisms of microbial proteins contributing to invasion of the blood-brain barrier. Cell Microbiol 3, 277-287.

Huang, S. H., Stins, M. F. \& Kim, K. S. (2000). Bacterial penetration across the blood-brain barrier during the development of neonatal meningitis. Microbes Infect 2, 1237-1244.

Jacques, M., Belanger, M., Roy, G. \& Foiry, B. (1991). Adherence of Actinobacillus pleuropneumoniae to porcine tracheal epithelial cells and frozen lung sections. Vet Microbiol 27, 133-143.

Kielstein, P. \& Rapp-Gabrielson, V. J. (1992). Designation of 15 serovars of Haemophilus parasuis on the basis of immunodiffusion using heat-stable antigen extracts. J Clin Microbiol 30, 862-865.

Kielstein, P., Rosner, H. \& Muller, W. (1991). Typing of heat-stable soluble Haemophilus parasuis antigen by means of agargel precipitation and the dot-blot procedure. Zentralbl Veterinarmed B 38, 315-320.

Kniesel, U. \& Wolburg, H. (2000). Tight junctions of the blood-brain barrier. Cell Mol Neurobiol 20, 57-76.

Lichtensteiger, C. A. \& Vimr, E. R. (1997). Neuraminidase (sialidase) activity of Haemophilus parasuis. FEMS Microbiol Lett 152, 269-274.

Moller, K. \& Kilian, M. (1990). V factor-dependent members of the family Pasteurellaceae in the porcine upper respiratory tract. J Clin Microbiol 28, 2711-2716.

Morozumi, T. \& Nicolet, J. (1986a). Some antigenic properties of Haemophilus parasuis and a proposal for serological classification. J Clin Microbiol 23, 1022-1025.

Morozumi, T. \& Nicolet, J. (1986b). Morphological variations of Haemophilus parasuis strains. J Clin Microbiol 23, 138-142.

Munch, S., Grund, S. \& Kruger, M. (1992). Fimbriae and membranes on Haemophilus parasuis. Zentralbl Veterinarmed B 39, 59-64.

Oliveira, S. \& Pijoan, C. (2004). Haemophilus parasuis: new trends on diagnosis, epidemiology and control. Vet Microbiol 99, 1-12.
Oliveira, S., Batista, L., Torremorell, M. \& Pijoan, C. (2001). Experimental colonization of piglets and gilts with systemic strains of Haemophilus parasuis and Streptococcus suis to prevent disease. Can J Vet Res 65, 161-167.

Patrick, D., Betts, J., Frey, E. A., Prameya, R., Dorovini-Zis, K. \& Finlay, B. B. (1992). Haemophilus influenzae lipopolysaccharide disrupts confluent monolayers of bovine brain endothelial cells via a serum-dependent cytotoxic pathway. J Infect Dis 165, 865-872.

Rafiee, M. \& Blackall, P. J. (2000). Establishment, validation and use of the Kielstein-Rapp-Gabrielson serotyping scheme for Haemophilus parasuis. Aust Vet J 78, 172-174.

Rapp-Gabrielson, V. J. \& Gabrielson, D. A. (1992). Prevalence of Haemophilus parasuis serovars among isolates from swine. Am J Vet Res 53, 659-664.

Rapp-Gabrielson, V. J., Gabrielson, D. A. \& Schamber, G. J. (1992). Comparative virulence of Haemophilus parasuis serovars 1 to 7 in guinea pigs. Am J Vet Res 53, 987-994.

Schaller, A., Kuhnert, P., de la Puente-Redondo, V. A., Nicolet, J. \& Frey, J. (2000). Apx toxins in Pasteurellaceae species from animals. Vet Microbiol 74, 365-376.

Segales, J., Domingo, M., Balasch, M., Solano, G. I. \& Pijoan, C. (1998). Ultrastructural study of alveolar macrophages infected in vitro with porcine reproductive and respiratory syndrome (PRRS) virus, with and without Haemophilus parasuis. J Comp Pathol 118, 231-243.

St Geme, J. W., 3rd \& Falkow, S. (1990). Haemophilus influenzae adheres to and enters cultured human epithelial cells. Infect Immun 58, 4036-4044.

Tadjine, M., Mittal, K. R., Bourdon, S. \& Gottschalk, M. (2004). Development of a new serological test for serotyping Haemophilus parasuis isolates and determination of their prevalence in North America. J Clin Microbiol 42, 839-840.

Teifel, M. \& Friedl, P. (1996). Establishment of the permanent microvascular endothelial cell line PBMEC/C1-2 from porcine brains. Exp Cell Res 228, 50-57.

Townsend, G. C. \& Scheld, W. M. (1995). In vitro models of the blood-brain barrier to study bacterial meningitis. Trends Microbiol 3, 441-445.

Tunkel, A. R., Rosser, S. W., Hansen, E. J. \& Scheld, W. M. (1991). Blood-brain barrier alterations in bacterial meningitis: development of an in vitro model and observations on the effects of lipopolysaccharide. In Vitro Cell Dev Biol 27A, 113-120.

Tuomanen, E. (1996). Entry of pathogens into the central nervous system. FEMS Microbiol Rev 18, 289-299.

Turni, C. \& Blackall, P. J. (2005). Comparison of the indirect haemagglutination and gel diffusion test for serotyping Haemophilus parasuis. Vet Microbiol 106, 145-151.

Vahle, J. L., Haynes, J. S. \& Andrews, J. J. (1997). Interaction of Haemophilus parasuis with nasal and tracheal mucosa following intranasal inoculation of cesarean derived colostrum deprived (CDCD) swine. Can J Vet Res 61, 200-206.

Vanier, G., Segura, M., Friedl, P., Lacouture, S. \& Gottschalk, M. (2004). Invasion of porcine brain microvascular endothelial cells by Streptococcus suis serotype 2. Infect Immun 72, 1441-1449.

Virji, M., Kayhty, H., Ferguson, D. J., Alexandrescu, C. \& Moxon, E. R. (1991). Interactions of Haemophilus influenzae with cultured human endothelial cells. Microb Pathog 10, 231-245.

Zucker, B. A., Baghian, A., Traux, R., O'Reilly, K. L. \& Storz, J. (1996). Detection of strain-specific antigenic epitopes on the lipooligosaccharide of Haemophilus parasuis by use of monoclonal and polyclonal antibodies. Am J Vet Res 57, 63-67. 